\title{
Microscopy, Fluorescence, and Confocal Raman Imaging of Biotinylated Single- Walled Carbon Nanotubes Bound to Breast Tumor Cells
}

\author{
I.H. Musselman, ${ }^{1,2}$ P. Bajaj, ${ }^{1}$ C. Mikoryak, ${ }^{3}$ R.H. Wang, ${ }^{3}$ D.K. Bushdiecker II, ${ }^{1}$ P. Memon, ${ }^{1}$ G.R. \\ Dieckmann, ${ }^{1,2}$ R.K. Draper, ${ }^{1,2,3}$ and P. Pantano ${ }^{1,2}$ \\ ${ }^{1}$ Department of Chemistry, ${ }^{2}$ Alan G. MacDiarmid NanoTech Institute, ${ }^{3}$ Department of Molecular \\ and Cell Biology, The University of Texas at Dallas, Richardson, TX 75080
}

Surface functionalization of carbon nanotubes is a strategy for the synthesis of SWNT-targeting moiety constructs and allows these hybrid nanotube-conjugates to be useful in various biomedical applications [1-2]. In this work, carboxylated single-walled carbon nanotubes (C-SWNTs), chemically modified by nitric acid reflux, were covalently coupled to a tether of $(+)$-Biotinyl-3,6,9trioxaundecanediamine (biotin-LC-PEO-amine) using 1-ethyl-3-(3-dimethylaminopropyl) carbodiimide hydrochloride (EDC). The SWNT-(biotin-LC-PEO) amine constructs (B-SWNTs) were characterized by microscopic and spectroscopic methods. A sensitive and versatile sandwich immunoassay design was then developed that takes advantage of the specific interaction between Her2 receptors on breast tumor cells (BT-474 cell line) and a monoclonal antibody (Her-66) specific for the receptor. A biotinylated secondary antibody that recognizes the primary antibody was used to tag a NeutrAvidin ${ }^{\mathrm{TM}}$-FITC marker via the strong binding affinity between biotin and avidin. BSWNTs were then targeted specifically to bind available sites on the NeutrAvidin ${ }^{\mathrm{TM}}$-FITC. The surface distribution of receptors on BT-474 cells, indirectly marked by NeutrAvidin ${ }^{\mathrm{TM}}$-FITC bound to the antibody, was studied by immunofluorescence microscopy at 15 and $37^{\circ} \mathrm{C}$. Confocal Raman imaging was used to probe the localization of SWNT binding to the tumor cells.

AFM revealed distinct biotinylated sites on the SWNT surface that were reinforced by TEM evidence of Streptavidin gold labeling (Figure 1). Immunofluorescence images showed that the Her2 receptors were primarily localized at the cell membrane surface after the immunoassay at 15 ${ }^{\circ} \mathrm{C}$. At $37{ }^{\circ} \mathrm{C}$, cross-linking between the primary antibody and the receptors resulted in clustering and patching, typical of sequestered receptors (Figure 2). Confocal Raman imaging provided labelfree SWNT G-band detection, validating the specific targeting of B-SWNTs to tumor cells. The control studies, for which the primary antibody was omitted, showed insignificant binding of the BSWNTs (Figure 3).

In summary, these results indicate the potential use of chemical functionalization in achieving binding specificity, and the directed recognition of tumor targets.

References

[1] Z. Liu et al., Nano Res. 2 (2009) 85.

[2] E. Katz et al., Chem. Phys. Chem. 5 (2004) 1084.

[3] This work was supported by the Human Frontier Science Program (GRD), U.S. Army (RKD, IHM, GRD, PP), the Robert A. Welch Foundation (PP), the Texas Higher Education Coordinating Board Advanced Technology Program (RKD) and the Center for Applied Biology at The University of Texas at Dallas. 


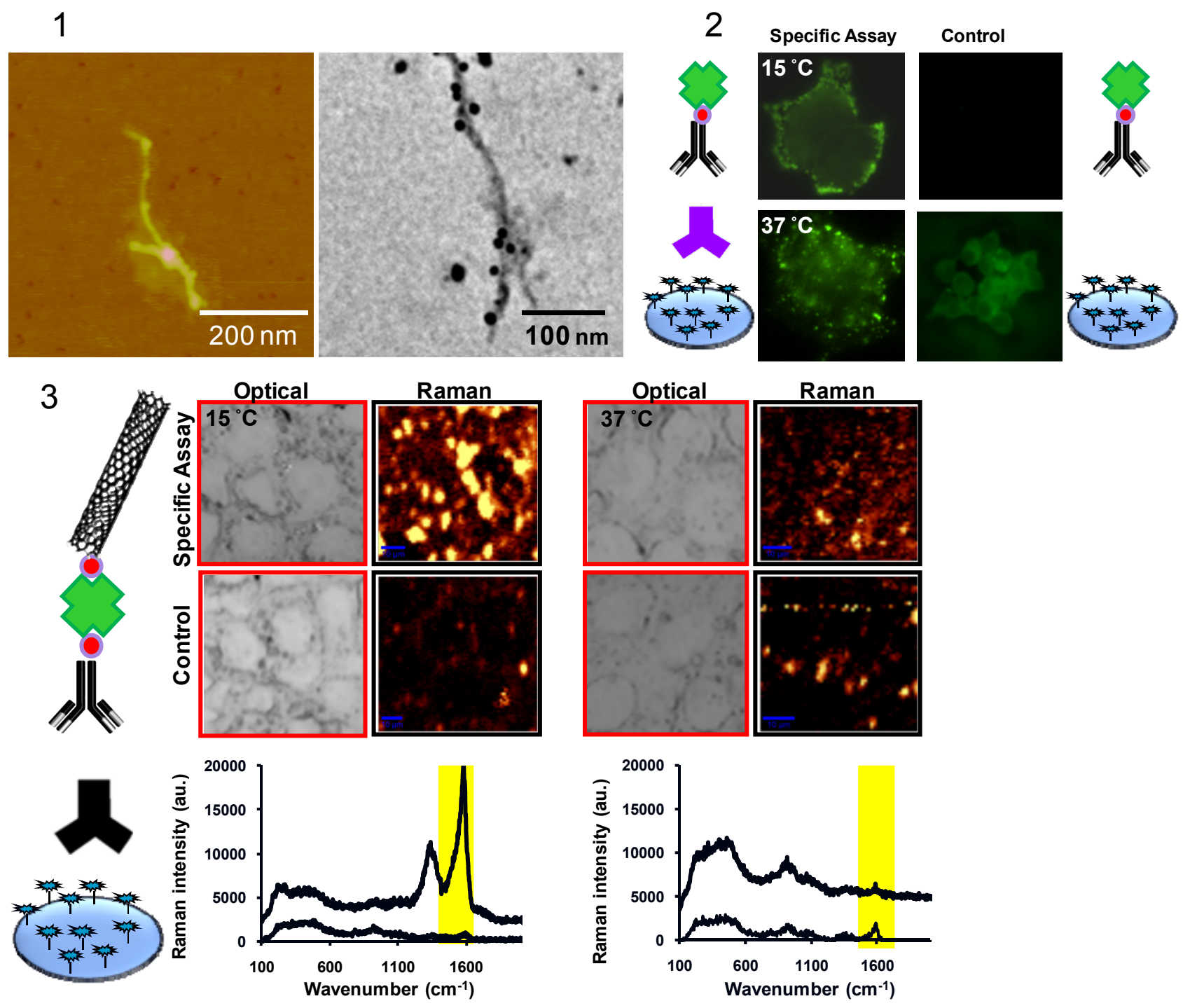

Figure 1. (left) AFM image $(800 \times 800 \mathrm{~nm})$ of B-SWNTs (solid white spheres denote the attachment of biotin LC-PEO-amine) and (right) TEM image of a Streptavidin gold-labeled SWNT-biotin LC-PEO-amine conjugate (solid black spheres mark the covalently coupled biotin sites on the SWNT).

Figure 2. (left) Immunofluorescence images (normalized to the same gray scale) showing the specific binding of NeutrAvidin ${ }^{\mathrm{TM}}$-FITC to Her2 receptors on BT-474 cells at 15 and $37{ }^{\circ} \mathrm{C}$ following the immunoassay format. (right) As control, the binding of NeutrAvidin ${ }^{\mathrm{TM}}$-FITC to the Her2 receptors in the absence of primary antibody is shown at 15 and $37^{\circ} \mathrm{C}$.

Figure 3. Optical images of BT-474 cell clusters $(70 \times 70 \mu \mathrm{m})$ and corresponding confocal Raman images $\left(0.3 \mathrm{~s}\right.$ integration time, $1 \mu \mathrm{m}$ per pixel) obtained from Raman spectra integrated from $1480-1660 \mathrm{~cm}^{-1}$ from the specific binding assay with B-SWNTs in the presence of primary antibody and without primary antibody as control at $15{ }^{\circ} \mathrm{C}$ (top left) and $37{ }^{\circ} \mathrm{C}$ (top right). The high intensity areas in the Raman images correspond to high G-band signal. The Raman spectra (bottom left, overlayed for clarity) from a bright spot in the Raman image show the characteristic G-band signature of carbon nanotubes bound to the BT-474 cells following the binding assay with B-SWNTs at $15{ }^{\circ} \mathrm{C}$. This G-band signal is mostly absent for the control sample (bottom left) as well as for the assay at $37^{\circ} \mathrm{C}$ (bottom right). 